\title{
Summary of Miniature NMR development
}

The effort in this project has been in 3 distinct directions. 1) First, we focused on development of miniature microfabricated micro-coil NMR detectors with maximum Signal-to-Noise (SNR) ratio. 2) Secondly, we focused on design of miniature micro-coil NMR detectors that have minimal effect on the NMR spectrum distortions. 3) Lastly we focused on the development of a permanent magnet capable of generating fields on the order of 1 Tesla with better than 10 ppm uniformity over

\section{Development of miniature micro-coil NMR detectors with maximal SNR}

To analyze SNR of coils it is very important to estimate accurately RF losses in coil conductors. The main difficulty in computing RF losses is related to highly inhomogeneous distribution of RF current in conductors. However, we have found an approximate, but highly accurate way to circumvent this difficulty by considering upper bounds on the RF eddy current losses. Based on this approach, we have developed a special mathematical method that can be used for RF loss analysis that is based only on calculations of DC currents and fields in micro-coils. This method is presented in:

G. Friedman, I.D. Mayergoyz, "On the estimate of eddy current losses in current carrying conductors", J. Appl. Phys., Vol. 87, No. 9, pp. 6926-6928, May 2000

Three types of micro-coil NMR detectors have been considered. They are multi-layer wound micro-wire coils, multi-layer scroll coils and planar spiral micro-coils. Multi-layer coils are harder to micro-fabricate, but have greater SNR. We have investigated SNR of such multi-layer coils theoretically and experimentally. These results are presented in:

S. Grant ${ }^{*}$, L. Murphy ${ }^{*}$, R.L. Magin and G. Friedman, "Analysis of multi-layer radio frequency microcoils for Nuclear Magnetic Resonance spectroscopy", IEEE Trans. Mag., vol. 37, no. 4, part II, pp. 2989-2998, July 2001

Grant", S.C.; Blackband, S.J.; Webb, A.G.; Friedman, G.; Magin, R.L.“APPLICATION OF RF MICROCOILS TO THE ANALYSIS OF SINGLE CELLS VIA MR

MICROSCOPY" Microtechnologies in Medicine and Biology, 1st Annual International, Conference On. 2000 , 2000 Page(s): $101-105$

Gimi, B.; Grant*, S.; Friedman, G.; Magin, R. "INVESTIGATION OF NMR SIGNALTO-NOISE RATIO FOR RF SCROLL MICROCOILS” Microtechnologies in Medicine and Biology, 1st Annual International, Conference On. 2000 , 2000 Page(s): 88 -91

Grant, Samuel*; Friedman, Gary; Growney, Eric*; Magin, Richard;

"SIGNAL-TO-NOISE IMPROVEMENT WITH MULTILAYERED RF

MICROCOILS", The $40^{\text {th }}$ Experimental Nuclear Magnetic Resonance Conference, Orlando, FL. February 28 - March 5, 1999. 
In summary, our investigations show that multi-layer coils can improve SNR over singlelayer coils and can be applied to such SNR sensitive applications as single cell NMR microscopy when the conductor thickness is 2-3 times the skin depth at the frequency of operation.

Sam Grant, Lisa Murphy and Barjor Gimi were the graduate students involved in the above research. Sam Grant has defended his PhD on the topic of "MR Microscopy of Isolated Single Cells with RF microcoils" and is currently a post-doctoral assistant at the University of Florida. Lisa Murphy defended her Master's Thesis on the topic of Finite Element Modeling of RF losses in multi-layer NMR coils and is currently at Agilant Corporation. Barjor Gimi is currently a PhD students working on applications of scroll micro-coils.

We have also investigated theoretically and experimentally planar micro-coils. Microfabrication of such coils is easier and more reliable than microfabrication of multiplayer coils, and, therefore, such coils may offer some advantages despite lower SNR values. In summary we have developed design method based on the special method we developed for RF loss estimation. We have also proved experimentally that such coils do provide reasonably high SNR. The results appear in:

S. Eroglu", G. Friedman, R.L. Magin "Estimate of losses and signal-to-noise ratio in planar inductive micro-coil detectors used for NMR", IEEE Trans. Mag., Vol. 37, no. 4, part I, pp. 2787-2789, July 2001

Eroglu*, S.; Grant", S.C.; Friedman, G.; Magin, P.L. "INVESTIGATION OF RF SPIRAL SURFACE COILS FOR NMR SPECTROSCOPY" Microtechnologies in Medicine and Biology, 1st Annual International, Conference On. 2000, 2000 Page(s): 92 -96

The lead graduate student on the project involving planar micro-coils is Sertac Eroglu, who is currently working toward his $\mathrm{PhD}$.

Development of methods for prediction of NMR spectrum distortions by micro-coils

One of the difficulties found when using micro-coils in NMR is in obtaining highresolution spectra. Part of the problem is that micro-coils introduce susceptibility mismatch very close to the sample and this, in turn, introduces static field inhomogeneity that are difficult to correct by shimming. In order to improve micro-coil designs we have developed highly accurate numerical method for prediction of NMR spectrum distortions caused by the micro-coils. This method is based on integral equation formulation that treats not the field itself as the unknown, but rather the field sources introduced due to the susceptibility mismatch. We have shown that this approach avoids the difficulties with accuracy of Finite Element methods. We have used the developed method to predict the NMR spectrum distortions due to multi-layer coils we have developed and compared these theoretical predictions with experiments. The agreement between experimental data and the theoretical prediction was remarkably accurate given that many simplifying assumptions were made. An example of the comparison is shown in Figure 1 below. 


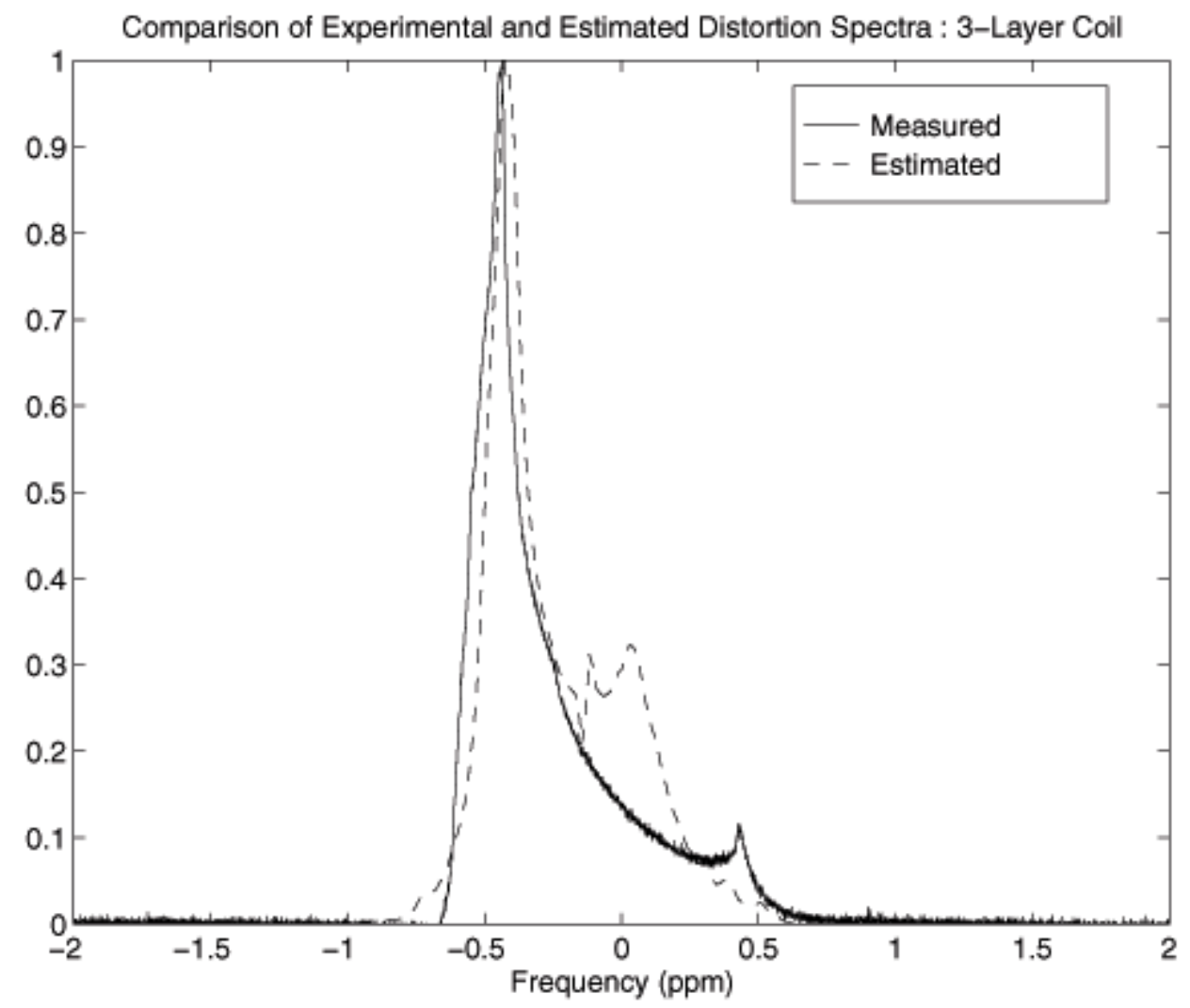

Figure 1. Comparison between calculated and measured NMR spectrum for a 3 layer micro-coil with a diameter and length of 850 micrometers.

The method is described in:

E. Growney*, G. Friedman, R. Gerald, "Computation of distortions in magnetic field and spectrum for NMR instruments", J. Appl. Phys., Vol. 85 No. 8, pp. 5205-5208, April 1999

Growney, Eric*; Friedman, Gary; Gerald II, Rex; Klingler, Robert;

Rathke, Jerome; Nunez, Luis; "EVALUATION AND DESIGN OF TOROID CAVITY

PROBES BASED ON CALCULATED DISTORTIONS", The $40^{\text {th }}$ Experimental

Nuclear Magnetic Resonance Conference, Orlando, FL. February 28 - March 5, 1999

Growney, E. ; Friedman, G.; Gerald II, R.; Klingler, R.; Rathke, J.;

"BOUNDARY ELEMENT TECHNIQUE FOR CALCULATIONS OF FIELD AND SPECTRAL DISTORTIONS", ISMRM Workshop on Computational Electromagnetics in Magnetic Resonance, College Station, TX. May 30 - June 1, 1998.

Growney, Eric *; Friedman, Gary; "COMPUTATIONS OF DISTORTIONS IN 


\section{MAGNETIC FIELD SPECTRUM FOR NUCLEAR MAGNETIC RESONANCE}

INSTRUMENTS", The 43rd Annual Conference on Magnetism and Magnetic Materials, Miami, FL. November 9-12,1998.

In addition a publication on the comparison of the numerical predictions of NMR spectrum distortions and experimental results for multi-layer micro-coils is currently under review in the Journal of Magnetic Resonance.

Eric Growney was the primary student on this part of the project. He defended his Master's Thesis and is currently with the Motorola Corporation.

\section{Development of the permanent magnet for miniature NMR instruments}

In this part of the project we have considered several different candidates for the permanent magnet design capable of delivering static magnetic field close to 1 Tesla and, at the same time, having a highly uniform (around $1 \mathrm{ppm}$ ) field in a volume of about 1 cubic millimeter. Ultimately we had settled on a magnet design illustrated in Figure 2 as a part of the overall miniature NMR system assembly.

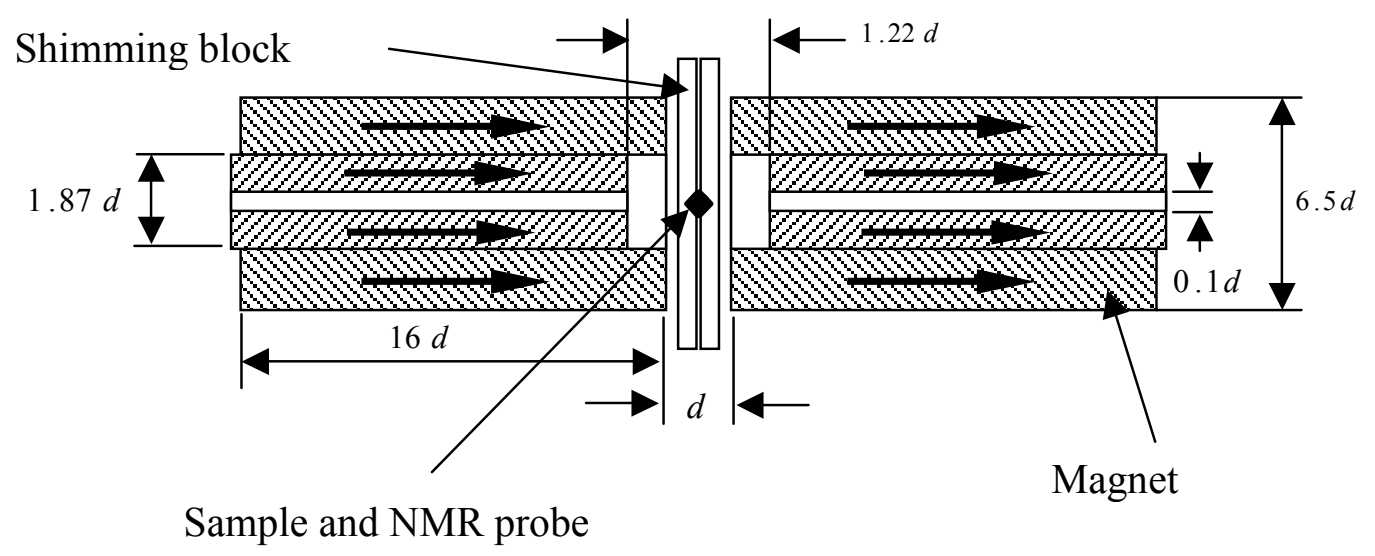

Figure 2. Permanent magnet as a part of miniature NMR instrument assembly.

The main idea of the above NMR magnet was to fabricate each of its two sides using two cylinrically shaped parts. The smaller cylinderical part was designed to fit tightly into the larger one, leaving the possibility of adjusting its axial position. The parts were fabricated by a company specializing in permanent magnet fabrication for $d=0.5$ centimeter. We attempted to put together the entire structure using aluminum guides at the University of Illinois at Chicago. This attempt, however, resulted in damage to the permanent magnet parts due to our own inexperience in dealing with assembly of brittle permanent magnets.

We did measure the field of the magnet and obtained about 0.8 Tesla reading from a Hall probe sensor inserted into the magnet's gap. However, this field was highly non-uniform (greater than 10,000 ppm) due to damage in the parts. 
In addition to the above magnet, a shimming block was designed and fabricated for the NMR instrument. This shimming block was designed to be operated with about 100 milliamper DC current source and consisted of multiple layers of printed circuit wires fabricated on thin flexible printed circuit substrates. However, the design was never tested because the magnet produced too large of a field variation to be shimmed by this shimming block. 